\title{
Micoquímica en México: breve reseña histórica
}

\author{
Mycochemistry in Mexico: brief historical overview
}

\author{
Jorge Suárez-Medellín ${ }^{1}$, César Espinoza², Guillermo Mendoza² , Ángel Trigos ${ }^{2}$ \\ ${ }^{1}$ Centro de Investigaciones Cerebrales, Universidad Veracruzana, Av. Luis Castelazo Ayala s/n, Col. Industrial Ánimas, 91190, Xalapa, \\ Veracruz, México. \\ ${ }^{2}$ Laboratorio de Alta Tecnología de Xalapa, Universidad Veracruzana. Médicos 5, Unidad del Bosque, 91010 , Xalapa, Veracruz, México.
}

\section{RESUMEN}

Antecedentes: En nuestro país, uno de los principales testigos del desarrollo de la química de hongos (a la que en lo sucesivo nos referiremos como micoquímica), ha sido el otrora llamado Boletín de la Sociedad Mexicana de Micología, posteriormente Revista Mexicana de Micología y hoy Scientia Fungorum.

Objetivo: Ofrecer un panorama general del desarrollo de la micoquímica mexicana a través del recuento de algunos de sus principales trabajos hasta la fecha.

Métodos: Se llevó a cabo una revisión bibliográfica de la literatura relacionada con la micoquímica producida en nuestro país, tomando en cuenta como fuente central de información los archivos de la revista Scientia Fungorum en sus distintas épocas (1968-2018), complementando lo anterior con estudios publicados por científicos mexicanos en las principales revistas indexadas en el área.

Resultados y conclusiones: Los trabajos de investigación consultados fueron agrupados de acuerdo a su disciplina e interés, para ello, se dividieron en cinco secciones: micotoxinas; estudios bromatológicos y afines; extractos crudos; aislamiento e identificación de metabolitos; y enzimas. Por lo anterior, la micoquímica en México se encuentra en su mejor momento, como lo atestigua la creciente cantidad y calidad de los trabajos publicados durante los últimos años.

Palabras clave: micoquímica, metabolitos fúngicos, productos naturales de hongos

\section{ABSTRACT}

Background: In our country, one of the main witnesses of the fungal chemistry development (hereafter referred to as mycochemistry), was the journal previously known as Boletín de la Sociedad Mexicana de Micología, subsequently Revista Mexicana de Micología, and today Scientia Fungorum.

Objective: Offer a brief overview about the development of Mexican Mycochemistry through the review of some of their main works up to this days.

Methods: An extensive review of all the available literature about mycochemistry produced in México was performed, using the archives of Scientia Fungorum (1968-2018) as primary source, and complementing with the works published by mexican scientists in the main indexed journals of the field.

Results and conclusions: The research works consulted were grouped according to their main subject, and then presented in five sections: mycotoxins; bromatologic studies and alike; raw extracts; isolation and identification of metabolites; and enzymes. The constant improvement in quantity and quality of the research published during the last years, is the best proof that the mexican mycochemistry is on its highest moment ever.

Keywords: mycochemistry, fungal metabolites, natural products derived from mushrooms

\section{ARTICLE HISTORY}

Received 18 July 2018 / Accepted 05 october 2018

On line 02 November 2018

\section{INTRODUCCIÓN}

La química de productos naturales y la micología tienen mucho más en común de lo que podría esperarse; ambas disciplinas han tenido un desarrollo impresionante a partir de la segunda mitad del siglo XX, y a pesar de ello, ambas siguen siendo erróneamente consideradas por los

\section{CORRESPONDING AUTHOR \\ « Ángel Trigos, atrigos@uv.mx \\ ORCID: 0000-0001-6112-2288}


El aislamiento, caracterización química y pruebas biológicas de los metabolitos producidos por hongos, forman parte del repertorio tradicional de temas estudiados por la química de productos naturales, bastando recordar tan sólo unos cuantos ejemplos históricos. En 1896, Gasio publicó detalles de un compuesto químico que resultó ser ácido micofenólico; en 1912, Alsberg estudió extractos de maíz infestado de moho; en 1929, Fleming descubrió la penicilina; durante los años treinta del siglo pasado, el grupo de Rastrick aisló y caracterizó cerca de 200 metabolitos producidos por miembros del reino Fungi (Trigos y Sambrano, 1992; Trigos, 1999).

En nuestro país, uno de los principales testigos del desarrollo de esta rama de la química de productos naturales (a la que por analogía nos referiremos a partir de ahora como micoquímica), ha sido el otrora llamado Boletín de la Sociedad Mexicana de Micología, posteriormente Revista Mexicana de Micología y hoy Scientia Fungorum. En ese sentido, resulta particularmente significativo que la primer conferencia impartida por el Dr. Teófilo Herrera en la entonces recién fundada Sociedad Mexicana de Micología en octubre de 1965, haya tratado, de manera indirecta, un tema intrínsecamente relacionado con la micoquímica: "Consideraciones sobre los hongos alucinógenos de México" (Herrera, 1968; Mata et al., 2008).

Por todo lo anterior, y sin mayor afán de ser una revisión exhaustiva, el presente trabajo pretende ofrecer un panorama general del desarrollo de la micoquímica mexicana a través del recuento de algunos de sus principales trabajos hasta la fecha. Para su realización, se llevó a cabo una revisión bibliográfica de la literatura relacionada con la micoquímica producida en nuestro país, tomando en cuenta como fuente central de información los archivos de la revista Scientia Fungorum en sus distintas épocas (1968-2018), pero también se incluyeron estudios publicados por científicos mexicanos en las principales revistas indexadas en el área. Los trabajos fueron agrupados de acuerdo a su enfoque de investigación y año de publicación.

\section{RESULTADOS Y DISCUSIÓN}

El término Micoquímica es muy amplio y abarca una gran variedad de temas, por lo que para facilitar la lectura de esta breve reseña histórica, se han agrupado en cinco secciones los estudios citados: micotoxinas, estudios bromatológicos y afines, extractos crudos, aislamiento e identificación de metabolitos y enzimas.

\section{Micotoxinas}

La capacidad que tienen muchos hongos microscópicos de producir diversos tipos de micotoxinas, fue una de las primeras preocupaciones de los micólogos mexicanos. De acuerdo con una revisión bibliográfica realizada por Campos-Nieto y Robledo (1979), el primer estudio sobre intoxicaciones de origen micótico llevado a cabo en México, fue el reportado en la tesis de licenciatura en medicina veterinaria de Dantes de la Vega en 1932. En dicho estudio, Dantes de la Vega usó un lote de 10 caballos, a 5 de los cuales se les suministró un alimento contaminado por Aspergillus glaucus, mientras que el resto tuvo acceso a alimentos libres de contaminantes. Previsiblemente, todos los individuos que recibieron el alimento contaminado, presentaron muestras de intoxicación después de 24 horas (citado por Campos-Nieto y Robledo, 1979).

Muchos de los trabajos pioneros consistían en la descripción de los efectos de micotoxicosis en distintos animales. Así, por ejemplo, Campos-Nieto et al. (1977) reportaron el aborto de un bisonte americano en el zoológico de Chapultepec, causado por el consumo de aflatoxinas. Por su parte, Moreno (1977) discute las condiciones requeridas por distintos mohos para colonizar granos almacenados, y relaciona los compuestos con el hongo que los produce, p. ej. aflatoxinas y Aspergillus flavus; esterigmatocistina y A. versicolor; así como patulina y Penicillium urticae; sin embargo, dicha información procede de literatura especializada previa y no de un estudio químico llevado a cabo en cepas mexicanas.

Campos-Nieto (1978), identificó la presencia de aflatoxina B1 en 33 fetos bovinos abortados, mediante pruebas de cromatografía en capa fina. Posteriormente, este mismo autor reportó dos casos de abortos porcinos causados por aflatoxicosis (Campos-Nieto et al., 1980). García-Aguirre y Martínez-Flores (1985) aislaron diferentes cepas de los géneros Aspergillus, Fusarium y Penicillium a partir de 90 muestras de maíz amarillo, representativas del maíz consumido en Ciudad de México durante el verano de 1983; posteriormente, cultivaron las cepas de Aspergillus spp. previamente aisladas y determinaron su capacidad de producir aflatoxinas mediante pruebas cromatográficas y bioensayos de toxicidad en larvas de Artemia salina. Algunos años 
después, estas mismas autoras, identificaron 12 especies pertenecientes al género Penicillium en muestras de maíz para consumo humano, todas ellas reportadas como productoras de micotoxinas (García-Aguirre y Martínez-Flores, 1991). Este mismo grupo de trabajo, analizó la presencia de aflatoxinas, zearalenona y ocratoxinas en cereales para el desayuno, comercializados en la Ciudad de México, y aunque no encontraron ninguno de los compuestos antes mencionados, pudieron aislar varias especies de mohos reportados como productoras de micotoxinas, especialmente pertenecientes a los géneros Aspergillus, Penicillium y Fusarium (García-Aguirre et al., 1999). En un estudio posterior, estas investigadoras analizaron 11 muestras de distintas marcas comerciales de mazapán en busca de aflatoxinas. Una vez más, no reportaron haber encontrado micotoxinas en ninguna de las muestras, pero identificaron la presencia de mohos pertenecientes a los géneros Aspergillus, Fusarium, Penicillium, Rhizopus y Absidia (García-Aguirre y Martínez-Flores, 2006). Esqueda Valle et al. (1995) reportaron la presencia de aflatoxina $\mathrm{M} 1$ en muestras de leche en polvo y pasteurizada, comercializada en Hermosillo, Sonora.

Carvajal et al. (2004) determinaron que la cantidad mínima de aflatoxina B1 capaz de inducir mutaciones en un modelo de Salmonella typhimurium es de $10 \mu \mathrm{g} / \mathrm{kg}$. Trigos et al. (2008) aislaron 24 cepas de hongos reportados como productores de micotoxinas a partir de frutas y hortalizas comercializadas en la ciudad de Xalapa, Veracruz. Posteriormente, Luna et al. (2010), aislaron 21 cepas Aspergillus niger a partir de muestras de café verde en condiciones de almacén, y determinaron su producción de ocratoxina A.

Díaz-Zaragoza et al. (2014), detectaron la presencia de aflatoxinas B1, B2, G1, G2, así como sus metabolitos hidroxilados y aflatoxicol, en pechugas de gallinas de postura.

Carvajal-Moreno (2015a), discutió el efecto de diversas micotoxinas, incluyendo a aflatoxinas, citreoviridina, moniliformina y fumonisinas, sobre la salud cardiovascular humana. Esta misma autora publicó una revisión de las principales características de la aflatoxina B1 como carcinógeno, así como algunas estrategias utilizadas para reducir sus riesgos en alimentos, y posteriormente analizó el papel de las aflatoxinas en el cáncer de sistema digestivo (Carvajal-Moreno 2015b, 2017).

Rosas-Contreras et al. (2016), analizaron identificaron y cuantificaron la presencia de aflatoxinas B1, B2, G1 y G2, en muestras de chile ancho, guajillo y piquín procedentes de México, Turquía y Sudáfrica; Carvajal-Domínguez et al. (2016), estudiaron estas mismas micotoxinas en salsas picantes industrializadas; Hernández-Camarillo et al. (2016) cuantificaron la presencia de aflatoxinas M1 y M2 en muestras de queso artesanal tipo Oaxaca en la ciudad de Veracruz; mientras que Garduño-García et al. (2017), cuantificaron el contenido de aflatoxina B1, B2, G1 y G2 en muestras de pimienta negra, blanca y verde provenientes de Egipto, Turquía, India y México.

\section{Estudios bromatológicos y afines}

A pesar de la tradición cultural del consumo de hongos comestibles silvestres en México, fue hasta 1995 que aparece el primer reporte de la composición proximal de diversos especímenes de hongos silvestres comestibles (Trigos et al., 1995a). Posteriormente, León-Guzmán et al. (1997) reportaron el análisis proximal, contenido de ácidos grasos y aminoácidos libres de los hongos comestibles silvestres Amanita rubescens, Boletus frostii, Lactarius indigo y Ramaria flava.

Soto-Velazco et al. (2005) llevaron a cabo un análisis proximal de los residuos de cosecha de Pleurotus columbinus y $P$. pulmonarius, y encontraron que su contenido de proteínas era de $14.4 \%$ y $8.98 \%$ respectivamente, contando ambas cepas con cantidades aceptables de aminoácidos esenciales tales como isoleucina, lisina, leucina y metionina, lo cual sugeriría su uso potencial como ingrediente en la formulación de alimentos.

Álvarez-Parrilla et al. (2007) determinaron la composición proximal, contenido de fenoles totales y actividad antioxidante de dos cepas comerciales de Agaricus bisporus, y tres cepas de hongos comestibles silvestres pertenecientes a los géneros Agaricus, Boletus y Macrolepiota.

López-Peña et al. (2013) reportaron el contenido total de proteínas, grasa cruda, cenizas y carbohidratos del micelio de dos cepas de Lentinula edodes, obtenidas en cultivo sumergido con suplementación de extractos de madera de vid (López-Peña et al., 2013) y González-Tijera et al. (2014) analizaron el perfil de ácidos grasos presentes en los cuerpos fructíferos de tres especies comestibles pertenecientes al género Pleurotus; mientras que Robles-García et al. (2016) reportaron el análisis proximal y la determinación de la capacidad antioxidante de Fistulinella wolfeana, utilizado como 
especie comestible en las comunidades otomíes de Tesquedó, Xajay y Tenasdá, en Amealco de Bonfil, Querétaro, México.

\section{Extractos crudos}

En la Micoquímica, el estudio de ciertas propiedades biológicas manifestadas por extractos crudos, da la pauta para motivar el estudio posterior de los metabolitos secundarios. Así, Muñoz y Dubovoy (1979a) estudiaron la capacidad antibiótica del basidiomicete Schizophyllum commune cultivado en diferentes medios y condiciones de $\mathrm{pH}$ y temperatura, y aunque no pudieron identificar a él o los metabolitos responsables de dicho efecto, reportaron su sospecha de que podía tratarse de un metabolito derivado de la vía del shikimato-corismato. Dichos autores, también notaron que la adición de cafeína al medio de cultivo de $S$. commune ocasionaba un decremento en su potencial antibiótico (Muñoz y Dubovoy, 1979b).

Por otro lado, Trigos et al. (2005a) estudiaron el efecto antibacteriano de los extractos acuosos de 14 cepas de hongos microscópicos aislados a partir del suelo y restos vegetales, encontrando que los extractos de Menisporopsis theobromae mostraban actividad inhibitoria contra Staphylococcus aureus, mientras que los extractos de Idriella sp. inhibían el crecimiento no solo de la cepa bacteriana antes mencionada, sino también de otras de Escherichia coli, Pseudomonas aeruginosa, Erwinia carotovora y Agrobacterium tumefaciens. Posteriormente, los compuestos responsables de dicho efecto fueron aislados e identificados, como se describe en Espinoza et al. (2008a). Este mismo grupo de trabajo investigó la actividad antifúngica de los extractos de cinco cepas pertenecientes a los géneros Ceratocystis, Curvularia, Idriella, Phytophthora y Rhizopus, encontrando que Idriella y Rhizopus presentan una actividad fungicida comparable con la del Captan ${ }^{\circledR}$ (Trigos et al., 2006; Espinoza et al., 2008b).

Mientras tanto, Valencia del Toro et al. (2008) investigaron la actividad inhibitoria de los extractos hexánicos de cuatro cepas de Pleurotus djamor contra las bacterias Bacillus subtilis, Staphylococcus aureus, Enterobacter agglomerans, Shigella dysenteriae, Yersinia enterocolitica, Klebsiella rhinoscleromatis y K. pneumoniae; así mismo, determinaron de manera general que dichos extractos contenían azúcares, flavonoides y sesquiterpenlactonas, pero no llevaron a cabo ningún proceso posterior de aislamiento e identificación de metabolitos a mayor profundidad.

Cinco años después, Leyva et al. (2013) estudiaron la fracción polar y no polar del extracto metanol:agua (70:30) de Phellinus merrillii, determinando su contenido de fenoles y flavonoides totales, así como su capacidad antioxidante y antibacteriana contra las cepas patógenas Salmonella enterica, Listeria monocytogenes, E. coli y $S$. aureus, mientras que Villanueva-Arce et al. (2013) reportaron el efecto inhibitorio de los extractos crudos concentrados de pigmentos del hongo Gibberella zeae (Fusarium graminearum), sobre las bacterias Salmonella typhi y S. aureus, pero no sobre B. subtilis, Shigella sp., E. coli, ni la levadura Candida albicans, y los hongos Colletotrichum acutatum, C. fragariae y C. gloeosporioides. De acuerdo con los autores de este estudio, el extracto crudo probablemente contenía fusarubina además de otros pigmentos afines, pero no llevaron a cabo ninguna identificación posterior de metabolitos a mayor profundidad.

Lagunes-Castro et al. (2015a) evaluaron el efecto del extracto cloroformo:metanol de Fusarium moniliforme sobre la producción de citocinas y su actividad citotóxica contra las líneas celulares Vero, MG-63, HBL-100, SW 1573, HeLa y WiDr. Este mismo grupo de trabajo, reportó la actividad inhibitoria de los extractos cloroformo:metanol de C. gloeosporioides y C. musae en contra de la bacteria E. coli, así como del extracto de IdrieIla lunata en contra de E. coli, S. aureus $\beta$-hemolítico y $P$. aeruginosa (Lagunes-Castro et al., 2015b).

López Sánchez et al. (2016) reportaron el efecto citotóxico de los extractos etanólicos de Agaricus xanthodermus, Boletus amygdalinus y Geastrum corollinum, sobre cultivos celulares de cáncer de pulmón H-460.

Meneses et al. (2016) investigaron el efecto de extractos hidroalcohólicos estandarizados de Ganoderma lucidum sobre parámetros bioquímicos del suero, contenido de grasa hepática, metabolismo del colesterol y composición de la microbiota intestinal, en ratones C57BL/6 alimentados con una dieta hipercolesterolémica, encontrando una reducción significativa en el colesterol sérico total, colesterol LDL, concentración de triglicéridos, colesterol hepático y triglicéridos hepáticos, asociada con una reducción en la expresión de genes lipogénicos.

Abriendo brecha en México con el estudio de hongos marinos, Couttolenc et al. (2016) reportaron el efecto 
antiproliferativo del extracto cloroformo:metanol del hongo endofítico marino Curvularia trifolii, en contra de las líneas celulares HBL-100, HeLa, SW 1573, T-47D y WiDr.

Islas-Santillán et al. (2017a) estudiaron la capacidad antioxidante y el contenido de polifenoles en extractos acuosos y etanólicos de Ganoderma brownii, G. applanatum y G. curtisii; mientras que, Adebayo et al. (2018) analizaron las propiedades antioxidantes, bacteriostáticas y bactericidas de extractos hidroalcohólicos estandarizados de Pleurotus levis, P. ostreatus, P. pulmonarius y $P$. tuber-regium.

\section{Aislamiento e identificación de metabolitos}

Entre los primeros trabajos específicamente micoquímicos realizados en nuestro país, hay que mencionar el estudio en el cual Domínguez et al. (1972) aislaron ergosterol y D-manitol a partir de Ganoderma lucidum, Fomes ignarius y F. badius originarios del estado de Nuevo León. Ese mismo grupo de trabajo reportó por primera vez el aislamiento de 1,4-dimetoxi-2-nitro-3,5,6-tricloro-benceno a partir de F. robiniae (Butruille y Domínguez, 1972). Guzmán et al. (1975) se refirieron a dichos trabajos como algunos de los "pocos estudios químicos" enfocados en hongos poliporoides. Como veremos más adelante, apenas cuatro décadas han bastado para cambiar radicalmente dicho panorama.

Ott et al. (1975) reportaron el intento de identificar ácido iboténico, psilocibina y bis-nori-angonina, por cromatografía en capa fina, en muestras de 11 licoperdáceos supuestamente alucinógenos. Ninguno de los hongos estudiados presentó cantidades apreciables de dichos metabolitos, ni tampoco efectos psicotrópicos en voluntarios. Sin embargo, Scleroderma verrucosum, una de las especies descritas, fue causante de una intoxicación gastrointestinal, sin que el metabolito responsable pudiese ser identificado. Ott (1975) también discute acerca de las características del uso recreativo de hongos alucinógenos en Estados Unidos y México en la época. Ott y Guzmán (1976) determinaron la presencia de psilocibina en varias especies pertenecientes a los géneros Psilocybe, Panaeolus y Psathyrella. Estos mismos autores, también identificaron la presencia de psilocibina y psilocina en muestras de Psilocybe stuntzii (Guzmán y Ott, 1976).

Repke et al. (1977) identificaron la presencia de baeocistina en diversas colecciones de Psilocybe, Conocybe y Panaeolus procedentes de Estados Unidos, Canadá, México y Perú; así mismo, demostraron la presencia de 4-hidroxitriptamina en $P$. baeocystis y $P$. cyanescens. Mundo Cansino y Ruíz-Herrera (1979) estudiaron la composición química de la pared celular de Phycomyces blakesleeanus y reportaron que está compuesta por quitina, quitosano, y dos poliurónidos distintos, además de proteínas, lípidos, fosfatos y azúcares neutros tales como fucosa, galactosa, glucosa y manosa. Posteriormente, Ramírez Chávez y Flores-Carreón (1991) estudiaron la composición química de las paredes celulares de dos cepas mutantes de este hongo, y encontraron diferencias en el contenido de azúcares totales y proteínas con respecto a la cepa silvestre.

Chinchilla et al. (1982), identificaron mediante análisis cromatográfico y bioensayos, la presencia de amanotoxinas $\alpha$ y $\beta$ en muestras de Amanita verna. Aroche y Fuentes (1982), por su parte, reportaron la presencia de ciclopéptidos tóxicos, incluyendo amanitinas $\alpha, \beta$ y $\gamma$ en los extractos metanólicos de Amanita phalloides, A. virosa y A. bisporigera. Posteriormente, Aroche et al. (1984) reportan la presencia de amanotoxinas y compuestos afines, identificados mediante el test de Meixner, cromatografía de capa fina y bioensayos, en distintas muestras de macromicetes silvestres de los municipios de Tenango del Aire y Chalco.

Sánchez et al. (1987) reportan la presencia de monómeros antraquinónicos como endocrocina, dermoluteina, dermorrubina, cinaluteina, cinarrubina, emodina, parietina, dermoglaucina, dermocybina (sic) y falacinol en seis especies pertenecientes al género Dermocybe y discuten su uso como marcadores quimiotaxonómicos. Ayer y Peña-Rodríguez (1987a), reportaron el aislamiento de fitotóxinas específicas del cultivo de canola a partir del hongo fitopatógeno Alternaria brassicae, las cuales fueron identificadas como destruxina B y desmetildestruxina $B$, ambas ya conocidas, así como homodestruxina B no descrita anteriormente. También, reportaron la producción de otros metabolitos, incluyendo los nuevos drimanos sesquiterpenos llamados desoxiuvidina B, albrassitriol e isoalbrassitriol, así como brassicadiol y un pentacétido prenilado $C_{15}$ (Ayer y Peña-Rodríguez, 1987b).

Pérez-Silva et al. (1988), mediante cromatografía en placa fina, identificaron la presencia de los pigmentos ácido cinabarínico y cinabarina en los cuerpos fructíferos de Pycnoporus sanguineus. 
Lappe et al. (1989) cuantificaron el contenido de etanol, ácido láctico y ácido acético en tejuino (una variedad de cerveza de maíz) producido por Saccharomyces cerevisiae y Geotrichum candidum, mediante cromatografía de gases.

Bandala-González y Trigos (1990) determinaron la presencia de alcaloides en diez especies de hongos de la zona del Cofre de Perote, Veracruz, previamente reportadas como comestibles. De igual forma, Trigos y Martínez-Carrera (1992) identificaron la presencia de ergosterol en $P$. ostreatus y posteriormente, Trigos reportó una serie de trabajos relacionados con la presencia de este metabolito, componente esencial de la membrana celular de los hongos y precursor sintético de la vitamina $D_{2}$, en una gran variedad de hongos silvestres y cultivados mexicanos, dentro del marco de un proyecto del CYTED con el ánimo de motivar la Química de Hongos en Latinoamérica, incluyendo diversas especies del género Pleurotus, Russula olivacea, e incluso en Phytophthora drechsleri, un oomicete a partir del cual dicho compuesto no había sido previamente aislado (Trigos y Franco, 1993; Trigos et al., 1994; Trigos et al., 1996a; Trigos et al., 1997a; Trigos et al., 1999; Trigos et al., 2005b).

Dada la ubicuidad del ergosterol en el reino Fungi, no resulta sorprendente la importancia de su estudio. En ese sentido, Trigos y Ortega Regules (2002) estudiaron la reacción química de foto oxidación del ergosterol a peróxido de ergosterol, como una estrategia alternativa contra las micosis, produciendo así la destrucción selectiva de hongos microscópicos patógenos. Años después, Trigos et al. (2011) estudiaron el papel de la macrosporina aislada a partir de Stemphylium lycopersici, en la formación de manchas necróticas causadas por este hongo fitopatógeno, basándose en la formación de oxígeno singulete, monitoreando éste, a través de la fotooxidación del ergosterol. Posteriormente, Lagunes y Trigos (2015) utilizaron dicha reacción de foto oxidación para detectar de manera indirecta el efecto fotosensibilizador de diversos compuestos antioxidantes de origen natural. Medina et al. (2015) estudiaron los mecanismos del daño oxidativo inducido por radicales peróxido al ergosterol en medio lípido, a través de modelos in silico; mientras que, Lagunes et al. (2017) con ésta misma fotooxidación sugieren el efecto prooxidante del vino tinto en presencia de Luz.

Por su parte, Gamboa-Angulo et al. (1997), aislaron metabolitos fitotóxicos a partir del cultivo de Alternaria solani, los cuales fueron identificados como zinniol, homozinniol y 6-[(3',3'-dimetilalil)oxi]-4-metoxi-5-metilftalida. Posteriormente, este grupo de trabajo aisló dos nuevos policétidos fitotóxicos llamados tagetolona y tagetenolona, además de tirosol y ácido p-hidroxibenzoico a partir del extracto crudo orgánico de filtrados de cultivo del hongo patógeno Alternaria tagetica. Complementariamente reportaron el aislamiento, síntesis y correlación estructura-actividad de derivados naturales de zinniol producidos por A. tagetica (Gamboa-Angulo et al., 2000; Gamboa-Angulo et al., 2001; Gamboa-Angulo et al., 2002).

Arana-López et al. (2003), aislaron peróxido de ergosterol y una mezcla compleja de triglicéridos a partir del micelio de A. tagetica. Moreno-Escobar et al. (2005), aislaron metabolitos que causan necrosis en hojas de papa, a partir de la purificación guiada de $A$. solani. Estas fitotoxinas fueron identificadas como 2-(2", $3^{\prime \prime}$-dimetil-but-1-enil)-zinniol, 8-zinniol metil éter, 5-(3',3'-dimetilaliloxi)-7-metoxi-6-metil-ftalida y 8-zinniol-2-(feniI)-etil éter.

Paralelamente a los trabajos antes mencionados, Trigos et al. iniciaron el aislamiento de dicetopiperacinas bioactivas a partir de cultivos miceliares de hongos fitopatogenos y así en 1995 reportaron el aislamiento de la ciclo-(L-prolil-L-glicina), la ciclo-(L-prolil-L-valina) y la ciclo-(L-leucil-L-prolina) a partir de Fusarium oxysporum. Un año después, aíslan por vez primera la dicetopiperazina denominada macrofominol a partir de Macrophomina phaseolina, y después identifican la presencia de ciclo-(L-prolil-L-glicina), ciclo-(L-prolil-Lvalina), ciclo-(L-leucil-L-prolina), ciclo-(L-tirosil-L-prolina) y ciclo-(L-alanil-L-prolina) en un cultivo de Pestalotia palmarum; así como, ciclo-(L-prolil-L-glicina), ciclo-(L-prolil-Lvalina), ciclo-(L-leucil-L-prolina), ciclo-(L-alanil-L-prolina) y ciclo-(L-fenilalanil-L-prolina) en C. gloeosporioides; y ciclo-(L-valil-L-prolina), ciclo-(L-leucil-L-prolina) y ciclo-(L-isoleucil-L-prolina) en Emericella rugulosa (Trigos et al., 1995b y c; Trigos et al., 1996b; Trigos et al., 1997b; Trigos et al., 2005c). Continuando con la química de hongos microscópicos, ese mismo grupo (2001) aisló el esterol fluorescente ergosta-4,6,8(14),22-tetraen-3-ona, además de ergosterol y cerevisterol a partir de Fusarium semitectum. Guzmán-López et al. (2007) reportaron el aislamiento de tirosol y triptofol a partir de $\mathrm{Ce}$ ratocystis adiposa. Al año siguiente, Espinoza et al. (2008a) pudieron aislar 5-hidroximetil-2-furaldehído 
y 1-n-butil- $\beta$-D-glucopiranósido a partir de extractos metanólicos del caldo de cultivo de Idriella sp. Ambos compuestos presentaron actividad inhibitoria in vitro en contra de las bacterias Xanthomonas axonopodis, Pectobacterium carotovorum, P. chrysanthemi y Erwinia amylovora.

Cruz-Cruz et al. (2009), establecieron una estrategia para purificar las fitotóxinas hidrofílicas presentes en el filtrado acuoso de Mycosphaerella fijiensis. Dos años más tarde, evaluaron los efectos fisiológicos de estás fitotoxinas hidrofílicas producidas por M. fijiensis, agente causal de la enfermedad sigatoka negra en plantas de banano (Cruz-Cruz et al., 2011).

Márquez-Fernández et al. (2013a) reportaron el aislamiento de ácido palmítico, ácido oléico, ácido esteárico y ácido linoléico a partir de los glomérulos de $C$. gloeosporioides cultivado en medio líquido. Este mismo grupo de trabajo, reportó el aislamiento de cuatro derivados del lanosterol a partir de Pisolithus arhizus, los cuales fueron identificados como 22-R-lanostan-8, 24(28)-dien-3 3 , 22-diol; 22-R-lanostan-28-metil-8, 24(28)-dien-3 $\beta$, 22-diol; pisolactona y 25-metil-pisolactona (Márquez-Fernández et al., 2013b).

Trigos et al. (2013) reportan el aislamiento de epi-cercosporina con actividad antiproliferativa en contra de cultivos celulares de las líneas HBL-100, HeLa, SW 1573 y WiDr, a partir de cultivos de Cercospora piaropi.

Por otro lado, Martín-Rodríguez et al. (2014) analizaron el perfil metabólico de los extractos de 75 hongos acuáticos aislados a partir de arrecifes, lagos salinos y manglares, en busca de metabolitos capaces de inhibir la comunicación entre células bacterianas conocidas como "quorum sensing". Entre los compuestos reportados en este estudio, destacan los siguientes: sespendol, lisofungina, ácido fusárico, N-acetil-O-preniltirosina, tricosetina, beauvericina, beauvericina $D$, emericelamida $A$, variecolorina $N$, verrucarina $B$ y penicitido $B$, entre otros.

Mientras que, a partir de un hongo marino, Espinoza et al. (2016) aislaron ergosterol, peróxido de ergosterol, cerevisterol y brefeldina A con efecto antiproliferativo sobre células tumorales, a partir del micelio cultivado de Curvularia trifolii.

Debido al potencial medicinal que representa el género Ganoderma, Trigos y Suárez-Medellín (2011) Ilevaron a cabo una revisión bibliográfica exhaustiva, en la que se describieron los metabolitos derivados del lanosterol aislados hasta ese momento a partir de diversas es- pecies de dicho género. Lo anterior, dio pauta a una serie de trabajos químicos relacionados con estos hongos medicinales. Así, Suárez-Medellín et al. (2014), aislaron ergosterol, acetato de ergosterol, ergosta-7,22-dien$3 \beta$-ol, peróxido de ergosterol, ergosta-7,22-dien-3-ona, cerevisterol, ácido ganodérico DM, lucidumol A, ganoderitriol $\mathrm{M}$ y ganoderiol $\mathrm{D}$ a partir de los basidiocarpos silvestres de una cepa mexicana de G. lucidum. Por su parte, Mendoza et al. (2015) estudiaron los carpóforos silvestres y el micelio cultivado en medio líquido de $G$. oerstedii. A partir de los cuerpos fructíferos aislaron cinco esteroles (ergosta-7,22-dien-3 $\beta$-ol, peróxido de ergosterol, ergosterol, cerevisterol, y ergosta-7,22-dien3-ona) y tres triterpenoides (ganodermanondiol, ácido ganodérico $\mathrm{Sz}$ y ganoderitriol M), mientras que a partir del micelio cultivado obtuvieron ergosterol, cerevisterol y acetato de ácido ganodérico Y.

Suárez-Medellín et al. (2016) realizaron un análisis de cribado virtual para determinar la capacidad de los derivados del lanosterol aislados a partir de diversas especies pertenecientes al género Ganoderma como ligandos específicos del receptor nuclear de vitamina $D$ humano. Este mismo grupo de trabajo, también llevó a cabo un análisis in silico para determinar cuáles de los metabolitos esteroideos de Ganoderma spp. podrían actuar como ligandos selectivos del receptor de andrógenos humano. Ambos estudios tenían como finalidad explorar algunos de los mecanismos que explicarían el efecto antiproliferativo reportado para este grupo de compuestos (Vidal-Limón et al., 2017).

Ramos-Ligonio et al. (2017) evaluaron el efecto proinflamatorio de la ergosta-7,22-dien-3-ona aislada a partir de los cuerpos fructíferos de $G$. oerstedii.

Islas-Santillán et al. (2017b) reportan el aislamiento de los esteroles previamente conocidos ergosterol, estelasterol, peróxido de ergosterol y $5 \alpha$-ergosta-7,22dien-3-ona, a partir de los basidiocarpos de G. curtisii y G. applanatum.

Finalmente, los estudios químicos de hongos comestibles con potencial terapéutico, se encuentran representados mediante los reportes de Ramos-Ligonio et al. (2012), sobre el efecto en contra de Trypanosoma cruzi del peróxido de ergosterol aislado a partir de $P$. ostreatus y la posterior demostración de que el acetato de peróxido de ergosterol, obtenido semisintéticamente a partir del metabolito antes mencionado, también posee actividad tripanocida (Lobato-Tapia et al., 2012), así como con el trabajo de Meza-Menchaca et 
al. (2015) acerca del efecto amebicida ejercido por el peróxido de ergosterol aislado a partir de P. ostreatus. Mata et al. (2014) analizaron la composición química de volátiles presentes en Lentinula edodes y L. boryanus, encontrando que los compuestos característicos del aroma de las especies antes mencionadas son 3-octanona y 3-octanol, respectivamente. Por su parte Márquez-Fernández et al. (2014) reportaron el aislamiento de ergosterol, peróxido de ergosterol, cerevisterol, $3 \beta, 5 \alpha, 6 \beta, 9 \alpha$-tetrahidroxiergosta-7,22-dieno y $3 \beta, 5 \alpha, 9 \alpha$-trihidroxiergosta-7,22-dien-6-ona a partir de una cepa comercial de Pleurotus sp.

\section{Enzimas}

Reyna y Ruíz-Herrera (1987) estudiaron el mecanismo de la foto-activación de la enzima quitina sintetasa presente en los esporangióforos de Phycomyces blakesleeanus. Por su parte, Ávalos Lozano y Flores-Carreón (1989) aislaron y caracterizaron una enzima glucoronosil transferasa involucrada en la biosíntesis de poliurónidos, a partir de extractos libres de células de $P$ blakesleeanus.

Chagolla et al. (1987) reportaron la presencia de enzimas quitinasas en el citosol, membranas y paredes celulares de Mucor rouxii. Hernández Delgadillo y Ruíz-Herrera (1989) estudiaron las propiedades de la quitina sintetasa aislada a partir de las subunidades $16 \mathrm{~S}$ de M. rouxii, mientras que Alvarado et al. (1989) aislaron una fracción proteolítica capaz de inactivar a la quitina sintetasa, a partir del citosol de ese mismo hongo.

Toriello et al. (1988) determinaron las actividades enzimáticas de cinco cepas de Histoplasma capsulatum en fase levaduriforme, encontrando en todas ellas las actividades de fosfatasa alcalina, esterasa lipasa C8, fosfatasa ácida y fosfoamidasa, así como lipasa C14 y leucina arilamidasa en cuatro de ellas.

Salmones y Mata (2005) estudiaron el efecto de la presencia de compuestos solubles de lignina y fenoles sobre la producción de la enzima lacasa en 30 cepas pertenecientes al género Pleurotus, cultivadas bajo distintas condiciones. Posteriormente, estos mismos autores estudiaron la relación entre la actividad in vitro de la enzima lacasa y el crecimiento micelial en 71 cepas de Pleurotus djamor (Salmones y Mata, 2015). Esta especie es particularmente interesante en nuestro país dada su amplia distribución (Salmones, 2017).
Murrieta Hernández et al. (2005) Ilevaron a cabo un análisis electroforético de proteínas totales e isoenzimas esterasas en seis cepas pertenecientes a tres especies del género Pleurotus, con el fin de proponer el uso de marcadores bioquímicos como una alternativa a las características morfológicas en la identificación taxonómica.

Márquez-Fernández et al. (2007) estudiaron el papel de la enzima fosfopantenil transferasa CfwA/NpgA en el metabolismo de las aflatoxinas en Aspergillus nidulans.

Castellanos-Moguel et al. (2008) estudiaron la relación entre la actividad de las proteasas y quitinasas de Paecilomyces fumosoroseus con su virulencia en contra de la mosquita blanca Trialeurodes vaporariorum.

Sánchez-Rosario et al. (2011) estudiaron la producción de fenol oxidasa en tres cepas de Scytalidium thermophilum y caracterizaron la enzima proveniente de la cepa más productiva, encontrando que se trataba de una hemoproteína glicosilada con 0.7 moles de Fe por mol de proteína.

Adame-García et al. (2011) estudiaron la variación isoenzimática de las esterasas, en relación con la patogenicidad de diversas cepas de Fusarium sp., asociadas a la pudrición de tallo y raíz de vainilla.

Flores-Giubi et al. (2014) aislaron una proteína inductora de necrosis identificada como p47f, a partir del secretoma de Phytophthora capsici.

Gutiérrez-Soto et al. (2015) analizaron la capacidad de producción de enzimas celulasas, xilanasas, pectinasas, amilasas y lacasas en 74 cepas de basidiomicetes macroscópicos, aisladas en diferentes ecotipos del noreste de México. Posteriormente, este mismo grupo de trabajo determinó la capacidad de Pycnoporus sanguineus, Trametes maxima, T. versicolor y T. hirsuta para producir celulasas, xilanasas y lacasas, y evaluó su potencial en la biotransformación de pasto buffel (Medina-González et al., 2016).

Orencio-Trejo et al. (2016) evaluaron la producción de celulasa, endoglucanasa, exoglucanasa, xilanasa, $\beta$-glucosidasa y $\beta$-xilosidasa en Talaromyces stollii.

\section{CONCLUSIONES}

Como hemos visto a través de este somero recorrido histórico, durante la segunda mitad del siglo XX y lo que va del XXI se logró el establecimiento en nuestro país de una floreciente tradición en el estudio de la 
micoquímica. Hoy por hoy, la disciplina se encuentra en su mejor momento, como lo atestigua la creciente cantidad y calidad de los trabajos publicados durante los últimos años; sin embargo, aún queda mucho por hacer, la proverbial biodiversidad de hongos mexicanos dista de haber sido totalmente estudiada, no ya digamos su capacidad de producir metabolitos secundarios útiles o nocivos para el hombre. Queda para las siguientes generaciones de especialistas en química y biotecnología de hongos, la responsabilidad de continuar con tan titánica tarea.

\section{LITERATURA CITADA}

Adame-García, J., Á. Trigos, L.G. Iglesias-Andreu, N. Flores-Estevez, M. Luna, 2011. Variaciones isoenzimática y patogénica de Fusarium spp. asociadas con la pudrición de tallo y raíz de vainilla. Tropical and Subtropical Agroecosystems 13: 299-306.

Adebayo, E.A., D. Martínez-Carrera, P. Morales, M. Sobal, H. Escudero, M.E. Meneses, A. Avila-Nava, I. Castillo, M. Bonilla, 2018. Comparative study of antioxidant and antibacterial properties of the edible mushrooms Pleurotus levis, $P$. ostreatus, P. pulmonarius and $P$. tuber-regium. International Journal of Food Science and Technology 53: 1316-1330. DOI:10.1111/ijfs.13712.

Alvarado, M.B., M. Pedraza, E. López-Romero, 1989. Estudio de una fracción proteolítica de Mucor rouxii que inactiva la quitina sintetasa. Revista Mexicana de Micología 5: 9-22.

Álvarez-Parrilla, E., L.A. de la Rosa, N.R. Martínez, G.A. González Aguilar, 2007. Total phenols and antioxidant activity of commercial and wild mushrooms from Chihuahua, Mexico. Ciencia y Tecnología Alimentaria 5 (5): 329-334. https://doi. org/10.1080/11358120709487708

Arana López, G., M. Gamboa Angulo, K. García Sosa, F. Escalante Erosa, L. Peña Rodríguez, 2003. A chemical study of the mycelium of Alternaria tagetica. Journal of the Mexican Chemical Society 47 (4): 303-306.

Aroche, R.M., J. Cifuentes, F. Lorea, P. Fuentes, J. Bonavides, H. Galicia, E. Menéndez, O. Aguilar, V. Valenzuela, 1984. Macromicetos tóxicos y comestibles de una región comunal del Valle de México, I. Boletín de la Sociedad Mexicana de Micología 19: 291-318.

Aroche, R.M., P. Fuentes, 1982. Presencia de ciclopéptidos tóxicos en algunas especies de la sección Phalloidae del género Amanita en México. Boletín de la Sociedad Mexicana de Micología 17: 187-195.

Ávalos Lozano, M.A., A. Flores-Carreón, 1989. Biosíntesis de poliurónidos en Phycomyces blakesleeanus: Caracterización de la glucoronosil transferasa. Revista Mexicana de Micología 5: 43-65.

Ayer, W.A., L.M. Peña-Rodríguez, 1987a. Metabolites produced by Alternaria brassicae, the black spot pathogen of canola. Part 1. The phytotoxic components. Journal of Natural Products 50(3): 400-407. DOI: 10.1021/np50051a010

Ayer, W.A., L.M. Peña-Rodríguez, 1987b. Metabolites produced by Alternaria brassicae, the black spot pathogen of canola. Part 2.
Sesquiterpenoid metabolites. Journal of Natural Products 50(3): 408-417. DOI: 10.1021/np50051a011

Bandala-González, E., A. Trigos-Landa, 1990. Determinación de alcaloides de algunas especies de hongos de la región del cofre de Perote, Veracruz, México. Micologia Neotropical Aplicada 3: 41-47.

Butruille, D., X.A. Domínguez, 1972. Un noveau produit naturel: Dimethoxy-1,4 Nitro-2 trichloro-3,5,6 Benzene. Tetrahedron Letters 3: 211-212. https://doi.org/10.1016/S0040-4039(01)84282-9

Campos-Nieto, E., 1978. Aborto micótico exógeno indirecto en bovinos de México. Boletín de la Sociedad Mexicana de Micología 12: 117-124.

Campos-Nieto, E., A. Cruz, J. Leyva, 1980. Dos casos de aborto porcino en una posible asociación con aflatoxina B1. Boletín de la Sociedad Mexicana de Micología 14: 101-105.

Campos-Nieto, E., J. Leyva, R. Pérez, 1977. Aborto producido por aflatoxinas en un bisonte del zoológico de Chapultepec, México, D.F. Boletín de la Sociedad Mexicana de Micología 11: $111-114$.

Campos-Nieto, E., E. Robledo, 1979. Los estudios sobre las aflatoxicosis animales en México. Boletín de la Sociedad Mexicana de Micología 13: 243-252.

Carvajal-Domínguez, H.G., M. Carvajal-Moreno, S. Ruíz-Velasco, M.T. Álvarez-Bañuelos, 2016. Presence of aflatoxins (mutagens and carcinogens) in industrialized chili sauces. Pharmaceutica Analytica Acta 7: 477. DOI:10.4172/2153-2435.1000477.

Carvajal-Moreno, M., 2015a. Mycotoxins that affect the human cardiovascular system. Pharmaceutica Analytica Acta 6: 365. DOI:10.4172/21532435.1000365.

Carvajal-Moreno, M., 2015b. Metabolic changes of aflatoxin B1 to become an active carcinogen and the control of this toxin. Immunome Research 11:104. DOI:10.4172/1745-7580.10000104.

Carvajal-Moreno, M. 2017. Do gastroenterologists consider aflatoxins as origin of digestive system cancers? Journal of Pharmacovigilance 5: 242. DOI:10.4172/2329-6887.1000242.

Carvajal, M., J.J. Espinosa-Aguirre, M.G. Moctezuma, M.E. Gonsebatt, F. Rojo, I. Pérez-López, 2004. Minimal amount of aflatoxin $b_{1}$ to produce a mutation in the Ames test with Salmonella thyphimurium Ta-98. Revista Mexicana de Micología 19: 71-79.

Castellanos-Moguel, R. Cruz-Camarillo, E. Aranda, T. Mier, C. TorieIlo, 2008. Relationship between protease and chitinase activity and the virulence of Paecilomyces fumosoroseus in Trialeurodes vaporariorum (Hemiptera: Aleyrodidae). Revista Mexicana de Micología 28: 71-80.

Chagolla, A., M. Pedraza, E. López Romero, 1987. Actividad quitinolítica en extractos libres de células miceliales de Mucor rouxii. Revista Mexicana de Micología 3: 283-292.

Chinchilla, E.F., R.M. Aroche, E. Pérez-Silva, P. Fuentes, 1982. Aspextos taxonómicos, químicos y farmacológicos de Amanita verna (agaricales). Boletín de la Sociedad Mexicana de Micología 17: 130-139.

Couttolenc, A., C. Espinoza, J.J. Fernández, M. Norte, G.B. Plata, J.M. Padrón, A. Shnyreva, Á. Trigos, 2016. Antiproliferative effect of extract from endophytic fungus Curvularia trifolii isolated from the "Veracruz Reef System" in Mexico. Pharmaceutical Biology 8: 1392-1397. DOI: 10.3109/13880209.2015.1081254 
Cruz-Cruz, C.A., K. García-Sosa, F. Escalante-Erosa, L.M. Peña-Rodríguez, 2009. Production of hydrophilic phytotoxins by Mycosphaerella fijiensis. Journal of General Plant Pathology 75: 191195. DOI: 10.1007/s10327-009-0165-1

Cruz-Cruz, C.A., K. García-Sosa, F. Escalante-Erosa, L.M. Peña-Rodríguez, 2011. Physiological effects of the hydrophilic phytotoxins produced by Mycosphaerella fijiensis, the causal agent of black sigatoka in banana plants. Journal of General Plant Pathology 77: 93-100. https://doi.org/10.1007/s10327-010-0288-4

Díaz-Zaragoza, M., M. Carvajal-Moreno, I. Méndez-Ramírez, N.C. Chilpa-Galván, E. Ávila-González, C.M. Flores-Ortiz, 2014. Aflatoxins, hydroxylated metabolites, and aflatoxicol from breast muscle of laying hens. Poultry Science 93: 3152-3162. DOI: 10.3382/ps.2014-04240

Domínguez. X.A., D. Butruille, A. Zamudio, C. Reyes, J. Castillo, 1972. Ergosterol et L-mannitol dans des champignons parasites. Phytochemistry 11: 2616. https://doi.org/10.1016/S00319422(00)88552-0

Espinoza, C., A. Couttolenc, J.J. Fernández, M. Norte, G.B. Plata, J.M. Padrón, A. Shnyreva, Á. Trigos, 2016. Brefeldin-A: an antiproliferative metabolite of the fungus Curvularia trifolii collected from the Veracruz Coral Reef System, Mexico. Journal of the Mexican Chemical Society 60(2): 79-82.

Espinoza, C., G. Viniegra-González, O. Loera, G. Heredia, Á. Trigos, 2008a. Antibacterial activity against plant pathogens by cruded extracts and compounds from Idriella sp. Revista Mexicana de Micología 26: 9-15.

Espinoza, C., G. Viniegra-González, O. Loera, B. Velásquez, Á. Trigos, 2008b. Antifungal activity of several fungi against plant pathogens. Micologia Aplicada International 20(2): 63-67.

Esqueda Valle, M., I. Higuera-Ciapara, J. Nieblas, 1995. Aflatoxina M1 en leche comercializada en Hermosillo, Sonora, México. Revista Mexicana de Micología 11: 179-183.

Flores-Giubi, M.E., M.A. Díaz-Brito, L. Brito-Argáez, K. García-Sosa, F. Escalante-Erosa, L.M. Peña-Rodríguez, I. Islas-Flores, 2014. Purification of $\mathrm{p} 47 \mathrm{f}$, a Necrosis-Inducing Protein Fraction from the Secretome of Phytophthora capsici. Journal of Phytopathology 162: 788-800. https://doi.org/10.1111/jph.12271

Gamboa-Angulo M.M., F. Alejos-González, L.M. Peña-Rodríguez, 1997. Homozinniol, a new phytotoxic metabolite from Alternaria solani. Journal of Agricultural and Food Chemistry 45(1): 282285. https://doi.org/10.1021/jf960134p

Gamboa-Angulo, M.M., F. Alejos-González, F. Escalante-Erosa, K. García-Sosa, G. Delgado-Lamas, L.M. Peña-Rodríguez, 2000. Novel dimeric metabolites from Alternaria tagetica. Journal of Natural Products 63(8): 1117-1120. DOI: 10.1021/np990422q

Gamboa-Angulo, M.M., F. Escalante-Erosa, K. García-Sosa, F. Alejos-González, G. Delgado-Lamas, L.M. Peña-Rodríguez, 2002. Natural zinniol derivatives from Alternaria tagetica. Isolation, synthesis, and structure-activity correlation. Journal of Agricultural and Food Chemistry 50(5): 1053-1058. DOI: 10.1021/ jf010641t

Gamboa-Angulo, M.M., K. García-Sosa,F. Alejos-González, F. Escalante-Erosa, G. Delgado-Lamas, L.M. Peña-Rodríguez, 2001. Tagetolone and tagetenolone: Two phytotoxic polyketides from
Alternaria tagetica. Journal of Agricultural and Food Chemistry 49(3): 1228-1232. DOI: 10.1021/jf000872k

García-Aguirre, G., R. Martínez-Flores, 1985. Aspergillus flavus y aflatoxinas en el maíz del Distrito Federal. Revista Mexicana de Micología 1: 189-199.

García-Aguirre, G., R. Martínez-Flores, 1991. Penicillium en maíz para consumo humano: problema de biodeterioro y riesgo micotoxigénico potencial. Revista Mexicana de Micología 7: 139-148.

García-Aguirre, G., R. Martínez-Flores, 2006. Mohos y aflatoxinas en mazapán. Revista Mexicana de Micología 23: 49-52.

García-Aguirre, G., R. Martínez-Flores, M.J. Hernández Del Ángel, 1999. Mohos y micotoxinas en cereales para desayuno: estudio preliminar. Revista Mexicana de Micología 15: 37-40.

Garduño-García, J.I., M. Carvajal-Moreno, F. Rojo-Callejas, S. Ruíz-Velasco, 2017. Detection of aflatoxins, mutagens and carcinogens in black, white and green peppers (Piper nigrum L.). Journal of Microbial and Biochemical Technology 9(3): 95-104. DOI: 10.4172/1948-5948.1000350

González-Tijera, M., O. Márquez-Fernández, M.R. Mendoza-López, G. Mata, Á. Trigos, 2014. A comparison of fatty acid content in three species of the genus Pleurotus. Revista Mexicana de Micología 39: 41-45.

Gutiérrez-Soto, G., G.E. Medina-González, J.E. Treviño-Ramirez, C.E. Hernández-Luna, 2015. Native macrofungi that produce lignin-modifying enzymes, cellulases, and xylanases with potential biotechnological applications. BioResources 10: 6676-6689.

Guzmán, G., J. Ott, 1976. Description and Chemical Analysis of a new species of hallucinogenic psilocybe from the Pacific Northwest. Mycologia 68(6): 1261-1267. DOI: 10.2307/3758762

Guzmán, G., R.G. Wasson, T. Herrera, 1975. Una iglesia dedicada al culto de un hongo, "Nuestro Señor del Honguito" en Chignahuapan, Puebla. Boletín de la Sociedad Mexicana de Micología 9: 137-147.

Guzmán-López, O., Á. Trigos, F.J. Fernández, M.J. Yañez-Morales, G. Saucedo-Castañeda, 2007. Tyrosol and tryptophol produced by Ceratocystis adiposa. World Journal of Microbiology and Biotechnology 23: 1473-1477. DOI: 10.1007/s11274-007-9392-9

Hernández-Camarillo, E., M. Carvajal-Moreno, V.J. Robles-Olvera, M. Vargas-Ortiz, M.A. Salgado-Cervantes, A.C. Roudot, G.C. Rodríguez-Jiménes, 2016. Quantifying the levels of the mutagenic, carcinogenic hydroxylated aflatoxins (AFM 1 and AFM 2) in artisanal oaxaca-type cheeses from the city of Veracruz, Mexico. Journal of Microbial and Biochemical Technology 8(6): 491-497. DOI: 10.4172/1948-5948.1000331

Hernández Delgadillo, R., J. Ruíz-Herrera, 1989. Propiedades de la quitina sintetasa presente en las subunidades 16 s de Mucor rouxii. Revista Mexicana de Micología 5: 23-41.

Herrera, T., 1968. Breve reseña histórica de la fundación de la Sociedad Mexicana de Micología. Boletín de la Sociedad Mexicana de Micología 1: 1-3.

Islas-Santillán, M.A., A. Castañeda Ovando, A. Álvarez Delgadillo, R. Valenzuela Garza, L. Romero-Bautista, J.M. Torres-Valencia, 2017a. Estudio preliminar de la actividad antioxidante de tres especies del género Ganoderma (Polyporaceae) nativas del estado de Hidalgo, México. Scientia Fungorum 46: 37-45. 
Islas-Santillán, M.A., L. Romero-Bautista, R. Valenzuela, J.M. Torres-Valencia, 2017b. Esteroles principlaes de Ganoderma curtisii y Ganoderma applanatum del estado de Hidalgo, México. Avances en Ciencia e Ingeniería 9(1): 43-54.

Lagunes, I., Á. Trigos, 2015. Photo-oxidation of ergosterol: Indirect detection of antioxidants photosensitizers or quenchers of singlet oxygen. Journal of Photochemistry and Photobiology 145: 30-34. DOI: 10.1016/j.jphotobiol.2015.02.014

Lagunes, I., F Vázquez-Ortega, Á Trigos, 2017. Singlet oxygen detection using red wine extracts as photosensitizers. Journal of Food Science 82 (9): 2051-2055. DOI: 10.1111/1750-3841.13815

Lagunes-Castro M.S., A. López Monteon, Á. Ramos-Ligonio, Á. Trigos, A. Salinas, C. Espinoza, 2015b. Actividad antibacteriana de extractos metanol:cloroformo de hongos fitopatógenos. Revista Mexicana de Fitopatología 33: 87-94.

Lagunes-Castro, M.S., A. Trigos, A. López-Monteon, G. Mendoza, A. Ramos-Ligonio, 2015a. Cytotoxic activity and induction of inflammatory mediators of the methanol:chloroform extract of Fusarium moniliforme. Revista Iberoamericana de Micología 32(4): 235-241. http://dx.doi.org/10.1016/j.riam.2014.11.005

Lappe, P., M. Ulloa, J. Gómez, 1989. Estudio microbiano y cromatográfico del tejuino de Jalisco, México. Revista Mexicana de Micología 5: 181-203.

León-Guzmán, M.F., I. Silva, M.G. López, 1997. Proximate chemical composition, free amino acid contents, and free fatty acid contents of some wild edible mushrooms from Querétaro, México. Journal of Agricultural and Food Chemistry 45: 4329-4332. DOI: 10.1021/jf970640u

Leyva, J.M., J.J. Pérez-Carlón, G.A. González-Aguilar, M. Esqueda, J.F. Ayala-Zavala, 2013. Funcionalidad antibacteriana y antioxidante de extractos hidroalcohólicos de Phellinus merrillii. Revista Mexicana de Micología 37: 11-17.

Lobato-Tapia, C., Á. Ramos-Ligonio, A. López-Monteon, H. Mansilla, A. Trigos, 2012. In vitro trypanocidal activity of ergosterol and cholesterol derivative compounds. Latin American Journal of Pharmacy 31(8): 1126-1131.

López-Peña, D. A. Gutiérrez, M. Esqueda, 2013. Cinética de crecimiento y composición química del micelio de Lentinula edodes cultivado en medio líquido suplementado con extractos de madera de vid. Revista Mexicana de Micología 37: 51-59.

López Sánchez, H., N. Ayala Sánchez, O. Rodríguez Alcantar, A. PortiIlo López, A. Íñiguez Martínez, I. Soria Mercado, 2016. Actividad citotóxica de extractos de hongos silvestres de Ensenada, Baja California, México. Revista Mexicana de Micología 43: 37-42.

Luna, M., Y. Lozada, Á. Trigos, 2010. Aislamiento de cepas de Aspergillus niger, productoras de ocratoxina $A$, en café verde (Coffea arabica) almacenado. Revista Mexicana de Micología 32: 63-68.

Márquez-Fernández, O., M. Cano, A. Salinas, O. Guzmán-López, C. Espinoza, Á. Trigos, 2013a. Composición química de glomérulos producidos por Colletotrichum gloeosporioides. Revista Mexicana de Micología 38: 2-7.

Márquez-Fernández, O., E. Herrera, O. Castellanos-Onorio, A.Estrada-Torres, Á. Trigos, 2013b. Identificación de un derivado de lanosterol procedente del hongo cultivado Pisolithus arhizus (scop.) Rauschert. Revista Chapingo Serie Ciencias Forestales y del Ambiente 19(2): 217-224. http://dx.doi.org/10.5154/r.rchscfa.2012.10.056

Márquez-Fernández, O., L.A. Juárez Pacheco, Á. Trigos, 2014. Aislamiento e identificación de esteroles de una cepa comercial de Pleurotus sp. Revista Chapingo Serie Ciencias Forestales y del Ambiente 20(2): 227-235. http://dx.doi.org/10.5154/r.rchscfa.2013.12.044

Márquez-Fernández, O., Á. Trigos, J.L. Ramos-Balderas, G. Viniegra-González, H.B, Deising, J. Aguirre, 2007. The phosphopantetheinyl transferase CfwA/NpgA is required for Aspergillus nidulans secondary metabolism and asexual development. Eukaryotic Cell 6: 710-720. DOI: 10.1128/EC.00362-06

Martín-Rodríguez, A.J., F. Reyes, J. Martín, J. Pérez-Yépez, M. León-Barrios, A. Couttolenc, C. Espinoza, Á. Trigos, V.S. Martín, M. Norte, J.J. Fernández, 2014. Inhibition of bacterial quorum sensing by extracts from aquatic fungi: first report from marine endophytes. Marine Drugs 12: 5503-5526. DOI: 10.3390/ md12115503

Mata, G., D. Salmones, R. Medel, R. Gaitán-Hernández, 2008. La Revista Mexicana de Micología en cifras: 40 años de trabajo editorial. Revista Mexicana de Micología 28: 1-6.

Mata, G., K., Valdez, R. Mendoza, Á. Trigos, 2014. HS/GC-MS analyzed chemical composition of the aroma of fruiting bodies of two species of genus Lentinus (higher basidiomycetes). Internacional Journal of Medicinal Mushrooms 16(5): 477-484. DOI: 10.1615/IntJMedMushrooms.v16.i5.60

Medina, M.E., C. Luga, Á. Trigos, 2015. Mechanism and kinetics of the oxidative damage to ergosterol induced by peroxyl radicals in lipid media: a theoretical quantum chemistry study. Journal of Physical Organic Chemistry 29: 196-203. https://doi. org/10.1002/poc.3520

Medina-González, G.E., H. Bernal Barragán, C.E. Hernández-Luna, C.A. Hernández-Martínez, G. Gutiérrez-Soto, 2016. Uso de basidiomicetos nativos en la biotransformación del pasto buffel (Cenchrus ciliaris) para mejorar la calidad nutricional. Revista Mexicana de Micología 43: 31-35.

Mendoza, G., J. Suárez-Medellín, C. Espinoza, A. Ramos-Ligonio, J. J. Fernández, M. Norte, Á. Trigos, 2015. Isolation and characterization of bioactive metabolites from fruiting bodies and mycelial culture of Ganoderma oerstedii (Higher Basidiomycetes) from Mexico. International Journal of Medicinal Mushrooms 17(6): 501-509. DOI: 10.1615/IntJMedMushrooms.v17.i6.10

Meneses, M.E., D. Martínez-Carrera, N. Torres, M. Sánchez-Tapia, M. Aguilar-López, P. Morales, M. Sobal, T. Bernabé, H. Escudero, O. Granados-Portillo, A.R. Tovar, 2016. Hypocholesterolemic properties and prebiotic effects of mexican Ganoderma lucidum in C57BL/6 mice. Plos One 11(7): e0159631. DOI:10.1371/journal. pone. 0159631.

Meza-Menchaca, T., J. Suárez-Medellín, C. Del Ángel-Piña, Á. Trigos, 2015. The amoebicidal effect of ergosterol peroxide isolated from Pleurotus ostreatus. Phytotherapy Research 29(12):1982-6. DOI: $10.1002 /$ ptr.5474.

Moreno, E., 1977. Los hongos y la calidad de los granos y semillas. Boletín de la Sociedad Mexicana de Micología 11: 127-135.

Moreno-Escobar J., A. Puc-Carrillo, M.C. Ceres-Farfán, L.M. Peña-Rodríguez, M. Gamboa-Angulo, 2005. Two new zinniol-relat- 
ed phytotoxins from Alternaria solani. Natural Product Research: Formerly Natural Product Letters 19(6): 603-607. https://doi.org $/ 10.1080 / 14786410410001729159$

Mundo Cansino, J., J. Ruíz-Herrera, 1979. Aislamiento, purificación y composición química de la pared celular de los esporangióforos de Phycomyces blakesleeanus. Boletín de la Sociedad Mexicana de Micología 13: 39-45.

Muñoz, A., C. Dubovoy, 1979a. Estudio fisiológico de la antibiosis de Schizophyllum commune. Boletín de la Sociedad Mexicana de Micología 13: 5-29.

Muñoz, A., C. Dubovoy, 1979b. Influencia de la cafeína en la antibiosis de Schizophyllum commune. Boletín de la Sociedad Mexicana de Micología 13: 30-37.

Murrieta Hernández, D.M., L. Iglesias Andreu, G. Mata, 2005. Caracterización bioquímica de seis cepas de Pleurotus. Revista Mexicana de Micología 21: 71-76.

Orencio-Trejo, M., J. Torres-Granados, A. Rangel-Lara, E. Beltrán-Guerrero, S. García-Aguilar, C. Moss-Acosta, H. Valenzuela-Soto, S. De la Torre-Zavala, A. Gastelum-Arellanes, A. Martínez, A. Tiessen, E. Díaz-Mireles, E. Lozoya-Gloria, 2016. Cellulase and xylanase production by the mexican strain Talaromyces stolii LV186 and its application in the saccharification of pretreated corn and sorghum stover. Bioenergy Research 9:1034-1045. https://doi.org/10.1007/s12155-016-9791-6

Ott, J., 1975. Notes on recreational use of hallucinogenic mushrooms. Boletín de la Sociedad Mexicana de Micología 9: 131135.

Ott, J., G. Guzmán, 1976. Detection of psilocybin in species of Psilocybe, Panaeolus and Psathyrella. Lloydia 39: 258-260.

Ott, J., G. Guzmán, J. Romano, J.L. Díaz, 1975. Nuevos datos sobre los supuestos licoperdáceos psicotropicos y dos casos de intoxicación provocados por hongos del género Scleroderma en México. Boletín de la Sociedad Mexicana de Micología 9: 67-76.

Pérez-Silva, E., E. Aguirre-Acosta, C. Pérez-Amador, 1988. Aspectos sobre el uso y la distribución de Pycnoporus sanguineus (polyporeaceae) en México. Revista Mexicana de Micología 4: 137-144.

Ramos-Ligonio, Á., A. López-Monteon, Á. Trigos, 2012. Trypanocidal activity of ergosterol peroxide from Pleurotus ostreatus. Phytotherapy Research 26: 938-943. https://doi.org/10.1002/ptr.3653

Ramos-Ligonio, Á., A. López-Monteon, M.S. Lagunes-Castro, J. Suárez-Medellín, C. Espinoza, G. Mendoza, Á. Trigos, 2017. In vitro expression of toll-like receptors and proinflammatory molecules induced by ergosta-7,22-dien-3-one isolated from a wild mexican strain of Ganoderma oerstedii (Agaricomycetes). International Journal of Medicinal Mushrooms 19(3): 203-211. DOI: 10.1615/IntJMedMushrooms.v19.i3.20

Ramírez Chávez, E., A. Flores-Carreón, 1991. Composición química de las paredes celulares de las esporas de las mutantes S-347 y S-377 de Phycomyces blakesleeanus. Revista Mexicana de Micología 7: 11-20.

Repke, D.B., D.T. Leslie, G. Guzmán, 1977. Baeocystin in Psilocibe, Conocybe and Paneolus. Lloydia 40(6): 566-578

Reyna, G., J. Ruíz-Herrera, 1987. Fotoactivación in vitro de la quitina sintetasa de los esorangióforos de Phycomyces blakesleeanus. Revista Mexicana de Micología 3: 249-263.
Robles-García, D., E. Yahia, J. García-Jiménez, E.U. Esquivel-Naranjo, F. Landeros, 2016. First ethnomycological record of Fistulinella wolfeana as an edible species and some of its nutritional values. Revista Mexicana de Micología 44: 31-39.

Rosas-Contreras, C., M. Carvajal-Moreno, F. Rojo-Callejas, S. Ruiz-Velasco, 2016. Identification and HPLC quantification of aflatoxins in dried chili peppers (Capsicum annum L.) in Mexico and other countries. Drug Metabolism \& Toxicology 7(1): 198. DOI: 10.4172/2157-7609.1000198.

Salmones, D., 2017. Pleurotus djamor, un hongo con potencial aplicación biotecnológica para el neotrópico. Scientia Fungorum 46: 73-85.

Salmones, D., G. Mata, 2005. Efecto de la presencia de compuestos solubles de lignina y fenoles sobre la producción de lacasa y biomasa en cultivos de Pleurotus spp. Revista Mexicana de Micología 21: 63-69.

Salmones, D., G. Mata, 2015. Laccase production by Pleurotus djamor in agar media and during cultivation on wheat straw. Revista Mexicana de Micología 42: 17-23.

Sánchez Macías, E., E Pérez Silva, C. Pérez Amador, 1987. Consideraciones quimiotaxonómicas para el estudio de algunas especies del género Dermocybe (Cortinariaceae) en México. Revista Mexicana de Micología 3: 189-202.

Sánchez-Rosario, Y., J.E. Sánchez, R. Vázquez-Duhalt, R.H. Andrade-Gallegos, 2011. Producción y caracterización de la fenol oxidasa de Scytalidium thermophilum. Revista Mexicana de Micología 34: 31-42.

Soto-Velazco, C., J.C. Serratos, M. Ruíz López, P. García López, 2005. Análisis proximal y de aminoácidos de los residuos de cosecha del hongo Pleurotus spp. Revista Mexicana de Micología 21: 49-53.

Suárez-Medellín, J., G. Mendoza, C. Espinoza, M. Suárez-Quiroz, A. Trigos, 2014. Lanostanoids isolated from the basidiocarps of a mexican strain of the medicinal fungus Ganoderma lucidum (Curtis) P. Karst. Latin American Journal of Pharmacy 33(2): 224230.

Suárez-Medellín, J., T. Meza-Menchaca, J.A. Quiroz Carranza, Á. Trigos, A.M. Vidal-Limon, 2016. In silico analysis of lanostanoids characterized in Ganoderma mushrooms (Agaricomycetes) as potential ligands of the vitamin D receptor. International Journal of Medicinal Mushrooms 18(11): 1037-1047. DOI: 10.1615/IntJMedMushrooms.v18.i11.80

Toriello, C., T. Mier, E. Ojeda, M.R. Reyes Montes, F. Mariat, M.L. Taylor, 1988. Actividades enzimáticas en levaduras de Histoplasma capsulatum. Revista Mexicana de Micología 4: 275-280.

Trigos, Á., 1999. Química de los hongos. In: Rivera, A. (Ed.), Producción de vitamina D2 a partir de hongos macromicetos: Aspectos científicos, técnicos y económicos. CYTED-COLCIENCIAS, Bogotá. Pp. 19-61.

Trigos, Á., D. Bouyssounade, M. Sobal, P. Morales, 1996a. Ergosterol content in Pleurotus sajor-caju cultivated on diferent organic substrates. Micologia Neotropical Aplicada 9: 125-127.

Trigos, Á., G. Cancela, J.M. Ramos, 1999. Sterols from the wild fungus Russula olivácea. Micologia Neotropical Aplicada 12: 59-66.

Trigos, Á., O. Castellanos-Onorio, A. Salinas, M.J. Yáñez-Morales, 2005b. Ergosterol from Phytophthora drechsleri, a unusual me- 
tabolite of a member of this genus. Myocopathologia 159(3): 469-471. https://doi.org/10.1007/s11046-004-8156-4

Trigos, A., O. Castellanos-Onorio, A. Salinas, C. Espinoza, M.J. Yáñez-Morales, 2006. Antibiotic activity of several Phytopathogenic fungi. Micología Aplicada International 18(1): 3-6.

Trigos, Á., C. Espinoza, M. Martínez, O. Márquez, L.G. León, J.M. Padrón, M. Norte, J.J. Fernández, 2013. Antiproliferative activity of epi-cercosporin in human solid tumor cell lines. Natural Product Communications 8(2): 187-189.

Trigos, Á., C. Espinoza, L. Tovar, O. Guzmán, L. Cervantes, 2001. A fluorescent sterol from Fusarium semitectum. Micologia Aplicada International 13(2): 93-96.

Trigos, Á., G. Franco, 1993. Determinación de ergosterol en hongos silvestres mexicanos. Micologia Neotropical Aplicada 6: 105108.

Trigos, A., A. López-Malo, A. Estrada, 1995a. Proximate composition of wild species of edible mushrooms from Mexico. Micologia Neotropical Aplicada 8: 73-76.

Trigos, Á., L.C. Martínez, E.E. López-Reyes, 2005c. Diketopiperazines from cultures of the fungus Emericella rugulosa. Micologia Aplicada International 17 (1): 1-4.

Trigos, Á., D. Martínez-Carrera, 1992. Identificación de Ergosterol en Pleurotus ostreatus. Micologia Neotropical Aplicada 5: 11-15.

Trigos, Á., D. Martínez-Carrera, R. Hernández, M. Sobal, 1997a. Ergosterol content in fruit of Pleurotus ostreatus is variable. Micologia Neotropical Aplicada 10: 93-96.

Trigos, Á., G. Mendoza, M. Luna, G. Heredia, R.M. Arias, 2005a. Evaluación antibacteriana de hongos microscópicos del suelo y restos vegetales. Revista Mexicana de Micología 20: 89-92.

Trigos, Á., G. Mendoza, C. Espinoza, A. Salinas, J.J. Fernández, M. Norte, 2011. The role of macrosporin in necrotic spots. Phytochemistry Letters 4: 122-125. https://doi.org/10.1016/j.phytol.2010.12.007

Trigos, Á., K., Ramírez, A. Salinas, 2008. Presencia de hongos fitopatógenos en frutas y hortalizas y su relación en la seguridad alimentaria. Revista Mexicana de Micología 28: 125-129.

Trigos, Á., S. Reyna, L. Cervantes, 1995b. Three diketopiperazines from the cultivated fungus Fusarium oxysporum. Natural Product Letters 6:241-246. https://doi.org/10.1080/10575639508043166
Trigos, A., S. Reyna, B. Matamoros, 1995c. Macrophominol, a new diketopiperazine from cultures of Macrophomina phaseolina. Phytochemistry 40(6): 1697-1698. https://doi.org/10.1016/0031-9422(95)00626-I

Trigos, A., S. Reyna, G. Galindo, J.M. Ramos, 1996b. Diketopiperazines from cultures of Pestalotia palmarum. Natural Product Letters 8: 199-205. https://doi.org/10.1080/10575639608044894

Trigos, A., S. Reyna, M.L. Gutiérrez, M. Sánchez, 1997b. Diketopiperazines from cultures of the fungus Colletotrichum gloeosporioides. Natural Product Letters 11: 13-16. https://doi. org/10.1080/10575639708043751

Trigos, Á., N. Sambrano, 1992. ¿Nos habremos olvidado de los hongos? Educación Química 3(4): 290-297.

Trigos, Á., J. Suárez-Medellín, 2011. Biologically active metabolites of the genus Ganoderma: Three decades of myco-chemistry research. Revista Mexicana de Micología 34: 63-83.

Trigos, Á., A. Ortega-Regules, 2002. Selective destruction of microscopic fungi through photo-oxidation of ergosterol. Mycologia 94(4): 563-568. DOI: 10.2307/3761707

Trigos, Á., T. Zayas, L. Ortuño, M. Sobal, P. Morales, 1994. Contenido de ergosterol en algunas especies cultivadas de Pleurotus. Micologia Neotropical Aplicada 7: 43-46.

Valencia del Toro, G., M.E. Garín Aguilar, M.A. Téllez Jaimes, E. Durán Páramo, 2008. Actividad antibacteriana de extractos hexánicos de cepas de Pleurotus djamor. Revista Mexicana de Micología 28: 119-123.

Vidal-Limon, A.M., O.D., Luna-Martínez, F. Rojas-Durán, T. Meza-Menchaca, M.E. Hernández-Aguilar, Á. Trigos, J. Suárez-Medellín, 2017. Molecular dynamics and virtual screening analysis of lanosterol derivatives from Ganoderma medicinal mushrooms (Agaricomycetes) as selective ligands of human androgen receptor. International Journal of Medicinal Mushrooms 19(7): 595605. DOI: 10.1615/IntJMedMushrooms.2017021162

Villanueva-Arce, R., C.A. Aguilar-Pompa, Y.M Gómez y Gómez, G. Valencia-Del Toro, A.B. Piña-Guzmán, S. Bautista-Baños, 2013. Control de bacterias patógenas y hongos de postcosecha con extractos del pigmento de Gibberella zeae (Fusarium graminearum). Agrociencia 47: 691-705. 